\title{
NIC-CAGE: An Open-Source Software Package for Predicting Optimal Control Fields in Photo-Excited Chemical Systems
}

\author{
Akber Raza ${ }^{\mathrm{a}, \dagger}$, Chengkuan Hong ${ }^{\mathrm{b}, \dagger}$, Xian Wang ${ }^{\mathrm{c}}$, Anshuman Kumar ${ }^{\mathrm{d}}$, \\ Christian R. Shelton ${ }^{\mathrm{b}}$, Bryan M. Wong ${ }^{\mathrm{a}, \mathrm{c}, \mathrm{d}, \mathrm{e}, \mathrm{*}}$ \\ ${ }^{a}$ Department of Electrical $\&$ Computer Engineering \\ ${ }^{b}$ Department of Computer Science $\& 3$ Engineering \\ ${ }^{c}$ Department of Physics \& Astronomy \\ ${ }^{d}$ Materials Science ${ }^{c} 3$ Engineering Program \\ ${ }^{e}$ Department of Chemical $\& 3$ Environment Engineering and Department of Chemistry \\ University of California-Riverside, Riverside, California 92521, United States
}

\begin{abstract}
We present an open-source software package, NIC-CAGE (Novel Implementation of Constrained Calculations for Automated Generation of Excitations), for predicting quantum optimal control fields in photo-excited chemical systems. Our approach utilizes newly derived analytic gradients for maximizing the transition probability (based on a norm-conserving CrankNicolson propagation scheme) for driving a system from a known initial quantum state to another desired state. The NIC-CAGE code is written in the MATLAB and Python programming environments to aid in its readability and general accessibility to both users and practitioners. Throughout this work, we provide several examples and outputs on a variety of different potentials, propagation times, and user-defined parameters to demonstrate the robustness of the NIC-CAGE software package. As such, the use of this predictive tool by both experimentalists and theorists could lead to further advances in both understanding and controlling the dynamics of photo-excited systems.
\end{abstract}

Keywords: time-dependent Schrödinger equation; optimal quantum control; Crank-Nicolson; gradient ascent optimization

\footnotetext{
${ }^{*}$ Corresponding author.

E-mail address: bryan.wong@ucr.edu, Webpage: http://www.bmwong-group.com
} 


\section{PROGRAM SUMMARY}

Program Title: NIC-CAGE

Licensing provisions: GNU General Public License 3 (GPL)

Programming language: MATLAB or Python

Nature of problem: The NIC-CAGE software package predicts optimized (and constrained) electric fields that can drive a system from a known initial vibrational eigenstate to a specified final quantum state with a large $(\approx 1)$ transition probability.

Solution method: Analytic gradients, Crank-Nicolson propagation, and gradient ascent optimization

\section{Introduction}

The interaction between light and chemical/material systems is central to a multitude of technological applications, including energy-efficient photovoltaic materials, [1, 2, 3] plasmon-induced energy transfer, [4, 5, 6, 7] and sustainable photocatalysis. [8, 9, 10] Our capability to fully harness these light-induced systems has tremendous potential to grow further as we build our understanding on how to control the excited-state quantum dynamical processes in these systems. While the majority of theoretical and experimental studies have focused on how these systems interact with a given electromagnetic radiation source (i.e, a laser pulse or an external light source), fewer studies have investigated the inverse problem to ask "If we desire a specific behavior in a chemical/material system, can we construct a light source to achieve this, and what does its functional form look like?". [11, 12, 13.

To help address this "inverse" question, this work provides an opensource software package, NIC-CAGE (Novel Implementation of Constrained Calculations for Automated Generation of Excitations), for predicting optimized electric fields to control photo-excited chemical systems. With a precomputed electronic potential energy, $V(x)$ (which can be routinely obtained with most electronic structure software packages), the NIC-CAGE code calculates optimal electric fields, $\epsilon(t)$, that can drive a system from a known initial vibrational eigenstate to another desired state, as depicted in Fig. 1. Our approach utilizes newly derived analytic gradients to construct a control field, $\epsilon(t)$, that gives a large $(\approx 1)$ transition probability for (constrained) excitations from an initial to a specified final quantum state. The NIC-CAGE 
code is provided in the MATLAB and Python programming environments as two separate but fully self-contained implementations. We have chosen to implement this code in these high-level programming environments to allow general researchers to easily understand how these approaches and tools are used in practice to control photo-excited mechanisms in a variety of systems.

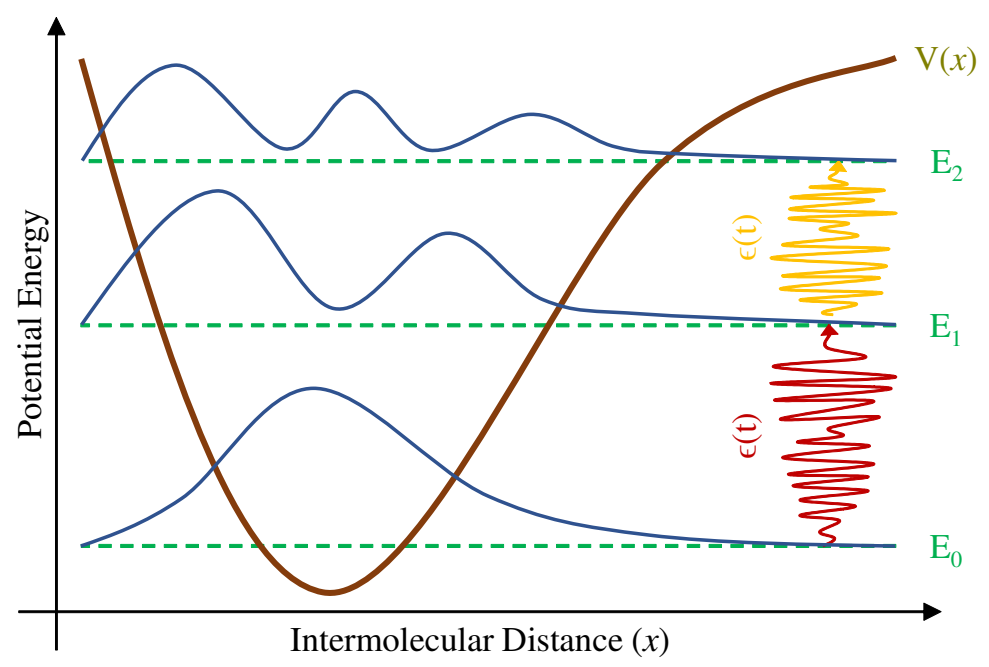

Figure 1: Schematic of an arbitrary potential energy well, $V(x)$, with various allowed energy levels $\left(\mathrm{E}_{0}, \mathrm{E}_{1}\right.$, and $\left.\mathrm{E}_{2}\right)$ and their associated probability densities, $|\psi(x)|^{2}$ (drawn in blue). The optimized control fields, $\epsilon(t)$, enable transitions between the desired vibrational eigenstates.

The present paper is organized as follows: Section 2 briefly outlines the basic theoretical concepts needed to understand the general idea for controlling the time evolution of a photo-excited system. Section 3 gives a more detailed description of our derivation and numerical implementation for carrying out this time-evolution operation. In particular, we provide analytic gradients for maximizing the transition probability based on a normconserving Crank-Nicolson propagation scheme, which have not been previously reported in the literature. With the basic concepts and mathematical formulation in place, Section 4 provides several examples and outputs on a variety of different potentials, propagation times, and user-defined parameters to demonstrate the robustness of the NIC-CAGE software package. Finally, Section 5 concludes with a brief discussion and perspective look at potential future applications of our approach. 


\section{Theory}

Within this section, we outline the basic theoretical concepts for optimizing/controlling the time evolution of a molecular system under the influence of electromagnetic radiation (i.e, a laser pulse or an external light source). A more detailed description of our numerical implementation and approach for carrying out this time-evolution optimization is given in Section 3. Although there have been previous studies on optimizing quantum control fields (each with their own purposes and advantages) [11, 12, 13, 14], we focus on the grid-based Crank-Nicolson discretization utilized in this work since (1) the analytic gradients for this norm-conserving approach have not been previously reported in the scientific literature, and (2) the resulting numerical scheme is more straightforward to implement and understand compared to other approaches.

The temporal dynamics for describing the motion of nuclei in a molecular system are governed by the time-dependent Schrödinger equation, which, in atomic units is given by:

$$
i \frac{\partial}{\partial t} \psi(x, t)=\mathcal{H}(x, t) \psi(x, t)
$$

where the time-dependent Hamiltonian operator, $\mathcal{H}(x, t)$, is

$$
\mathcal{H}(x, t)=-\frac{1}{2 m} \frac{\partial}{\partial x^{2}}+V(x, t) .
$$

In the expression above, $x$ denotes the reduced coordinate along a reaction path (cf. Fig. 1), $m$ is the effective mass associated with the molecular motion along the reaction path, and $V(x, t)$ is a time-dependent potential energy function (additional details on both $x$ and $V(x, t)$ are given later). The effective mass, $m$, is closely related to Wilson's G-matrix formalism [15], and previous work by us [16] gives details and an open-source code for calculating this molecular parameter along the reaction path.

The complex-valued molecular wavefunction, $\psi(x, t)$, in Eq. (1) represents the probability amplitude for the motion of the nuclei along the reduced coordinate path, and $|\psi(x, t)|^{2}$ can be interpreted as a probability density with the normalization condition

$$
\int_{-\infty}^{\infty}|\psi(x, t)|^{2} d x=1
$$


which holds for all $t$. In this work, we utilize the dipole approximation [5, 4, 17] in which the time-dependent potential can be written as

$$
V(x, t)=V(x)-\mu(x) \epsilon(t),
$$

where $V(x)$ is the time-independent portion of the potential that contains the Born-Oppenheimer electronic energy of the molecule along the reaction path (which can be obtained from a standard quantum chemistry calculation). The dipole moment function, $\mu(x)$, can also be readily computed from an electronic structure calculation at every point, $x$, along the reaction path as described in Ref. [18]. Finally, $\epsilon(t)$ is the time-dependent external electric field whose functional form is iteratively optimized using the NIC-CAGE software package in this work.

At this point, it is worth mentioning a few choices for the reduced coordinate, $x$, given in Eqs. (2) and (4), respectively. One of the most common choices for $x$ is the set of mass-weighted coordinates along the minimum energy path (also known as the intrinsic reaction coordinate), which is defined as the steepest descent path from a transition state toward reactants or products [19, 20]. However, obtaining this quantity can be computationally expensive since it typically requires a full computation of the molecular Hessian at each point along the reaction path. As such, prior work by us $[18,21,22,23,24,25]$ and other researchers [26, 27] have shown that a suitable path can be parameterized with a single internal coordinate such as a bond length [18], valence bend angle [23, 24, 25], or dihedral angle [21, 22] that can accurately describe reactions involving bond dissociation, isomerization, or internal rotation, respectively. Once a suitable reduced coordinate, $x$, is chosen, both $V(x)$ and $\mu(x)$ can be readily computed in most quantum chemistry packages such as Gaussian [28], Q-Chem [29], GAMESS [30], or NWChem [31] by carrying out a relaxed potential energy scan.

With $x$ and $V(x)$ properly chosen/computed, Eqs. (1) and (2) allow us to mathematically answer the question: "Given an electric field $\epsilon(t)$, how does an initial state, $\psi_{0}(x, t=0)$, evolve after some final time $T$ has elapsed?" However, as mentioned in the Introduction, we instead seek the answer to the following "inverse" question: "If we want to reach a desired final state $\psi_{N-1}(x, t=T)$ at time $T$ (after $N-1$ propagation steps), what does the functional form of $\epsilon(t)$ look like?" Providing accurate and efficient answers to this inverse question is the ultimate goal of the NIC-CAGE software package described in this work. To be more mathematically precise, we seek the functional form of an external electric field, $\epsilon(t)$, that maximizes the transition 
probability, $P\left[\psi_{N-1}(x)\right]$, given by

$$
P\left[\psi_{N-1}(x)\right]=\left|\int_{-\infty}^{\infty} \psi_{f}^{*}(x) \psi_{N-1}(x) d x\right|^{2},
$$

where $\psi_{f}$ is a known desired final target wavefunction (given by the user and discussed further below), and $\psi_{N-1}$ is obtained after applying $N-1$ succesive iterations. It should be noted that the transition probability is essentially a measure of the similarity of the final target and the propagated wavefunction. As written, the maximization of Eq. (5) is an unconstrained optimization problem to numerically solve for the time-dependent, external electric field $\epsilon(t)$. However, to make a direct connection to realistic experiments that may have limited power constraints for generating the optimal $\epsilon(t)$, we can augment Eq. (5) with a fluence penalty term:

$$
J\left[\psi_{N-1}(x), \epsilon\right]=P\left[\psi_{N-1}(x)\right]+F[\epsilon],
$$

where the fluence, $F[\epsilon]$, acts as a penalty to prevent unphysically large values of the electric field and is given by:

$$
F[\epsilon]=-\alpha \int_{0}^{T} \epsilon(t)^{2} d t
$$

where $\alpha$ is a positive constant to be chosen by the user. For the remainder of this paper, we focus on the constrained maximization of $J\left[\psi_{N-1}(x), \epsilon\right]$ given by Eq. (6).

With the quantum optimal control problem now properly defined, one can next choose the desired initial and final molecular wavefunctions to be used as initial conditions in the numerical quantum control procedure. In principle, both the initial and final wavefunctions can take on any form and are not even required to be stationary states of the Schrödinger equation (i.e., the numerical optimization techniques used in the NIC-CAGE software package can be used equally well for stationary or non-stationary states). In practice, however, one typically desires a control field that drives the system from a known initial vibrational eigenstate, $\psi_{i=\nu_{i}}(x)$, to another desired vibrational eigenstate, $\psi_{f=\nu_{f}}(x)$, of the system $\left(\nu_{i}\right.$ and $\nu_{f}$ denote the vibrational quantum numbers of the initial and final wavefunction, respectively). As such, to obtain these stationary wavefunctions as input, the NIC-CAGE software package contains a numerical routine for solving the time-independent 
Schrödinger equation; i.e., $\left[-\frac{1}{2 m} \frac{d \psi(x)}{d x^{2}}+V(x) \psi(x)=E_{i} \psi(x)\right]$ is diagonalized to obtain both $\psi_{i=\nu_{i}}(x)$ and $\psi_{f=\nu_{f}}(x)$. Put more concretely, we choose our initial state $\psi_{0}(x, t=0)$ to be a user-specified stationary vibrational eigenstate $\psi_{i=\nu_{i}}(x)$ of the time-independent Schrödinger equation, and the desired final state $\psi_{N-1}(x, t=T)$ is chosen to be a different vibrational eigenstate $\psi_{f=\nu_{f}}(x)$. To make our notation more succinct in the remainder of this paper, $\psi_{i=\nu_{i}}(x)$ and $\psi_{f=\nu_{f}}(x)$ are abbreviated as $\psi_{i}(x)$ and $\psi_{f}(x)$, respectively.

\section{Numerical Methodology}

With the basic theoretical concepts discussed in the previous section, we now give a detailed description of the various numerical approaches used in the NIC-CAGE code for calculating optimized control fields that drive the system from $\psi_{i}(x)$ to $\psi_{f}(x)$. Section 3.1 gives the numerical scheme used to discretize the time-independent portion of the Hamiltonian across a spatial grid. With the time-independent Hamiltonian properly discretized, Section 3.2 describes the Crank-Nicolson approach used in the NIC-CAGE code to numerically propagate the full time-dependent Schrödinger equation. Finally, Section 3.3 describes the gradient ascent approach for maximizing an objective functional of the transition probability $P$. Throughout the following subsections, we use the following notation: scalars are denoted by italic letters $(x)$, vectors by bold lower case letters (x) (unless specified otherwise), matrices by bold upper case letters $(\mathbf{X})$, the element in the $k^{\text {th }}$ row and $l^{\text {th }}$

column of matrix $\mathbf{X}$ by $[\mathbf{X}]_{k l}$, the identity matrix by $\mathbb{I}$, where $\mathbb{I}^{(m \pm)}$ denotes a matrix with entries of 1 on the $m$ th diagonal either above $(+)$ or below $(-)$ the main diagonal, a diagonal matrix having the entries $\mathbf{x}$ along the diagonal by $\operatorname{diag}(\mathbf{x})$, the Schur (element-wise) product by the $\odot$ symbol, and the conjugate and conjugate transpose of $\mathbf{X}$ by $\mathbf{X}^{*}$ and $\mathbf{X}^{\dagger}$, respectively.

\subsection{Grid-Based Discretization of the Time-Independent Hamiltonian}

We commence by discretizing the kinetic energy portion of the Hamiltonian operator across a grid of $L$ equidistant points on the interval $\left[x_{\min }, x_{\max }\right]$. Using a second-order five-point stencil finite difference approximation gives

$$
\begin{aligned}
& \frac{d^{2} \psi(x)}{d x^{2}} \approx \\
& \frac{-\psi(x-2 \Delta x)+16 \psi(x-\Delta x)-30 \psi(x)+16 \psi(x+\Delta x)-\psi(x+2 \Delta x)}{12(\Delta x)^{2}}
\end{aligned}
$$


where $\Delta x=\frac{x_{\max }-x_{\min }}{L-1}$. The notation of Eq. (8) can be considerably simplified by defining the $i$ th grid point as $x_{i}=x_{\min }+i \Delta x$ (where $i=0, \ldots, L-1$ ) and $\psi\left(x_{i}\right)=\psi^{i}$. With this simplified notation, the discretized time-independent Schrödinger equation can be written as

$$
\begin{aligned}
\mathcal{H}(x) \psi(x) & =-\frac{1}{2 m} \frac{\partial}{\partial x^{2}} \psi(x)+V(x) \psi(x) \\
& \approx-\frac{1}{2 m} \frac{-\psi^{i-2}+16 \psi^{i-1}-30 \psi^{i}+16 \psi^{i+1}-\psi^{i+2}}{12(\Delta x)^{2}}+V^{i} \psi^{i}
\end{aligned}
$$

The notation in Eq. (9) can be mathematically interpreted as a matrixvector equation, and the time-independent Hamiltonian can be written as the following matrix

$$
\mathbf{H}=-\frac{1}{24 m(\Delta x)^{2}}\left(-\mathbb{I}^{(2-)}+16 \mathbb{I}^{(1-)}-30 \mathbb{I}+16 \mathbb{I}^{(1+)}-\mathbb{I}^{(2+)}\right)+\mathbf{V}
$$

where $\mathbb{I}$ is an $L \times L$ identity matrix with entries of 1 on the main diagonal, $\mathbb{I}^{(1 \pm)}$ are $L \times L$ matrices with entries of 1 on the 1 st diagonal either above $(1+)$ or below $(1-)$ the main diagonal, $\mathbb{I}^{(2 \pm)}$ are $L \times L$ matrices with entries of 1 on the 2 nd diagonal either above $(2+)$ or below $(2-)$ the main diagonal, and $\mathbf{V}$ is an $L \times L$ diagonal matrix with entries $[\mathbf{V}]_{i j}=V\left(x_{i}\right) \delta_{i j}$, where $\delta_{i j}$ is the Kronecker delta. As mentioned in Section 2, the time-independent Hamiltonian matrix in Eq. (10) is diagonalized to obtain the initial and final states, $\psi_{0}(x, t=0)=\psi_{i}(x)$ and $\psi_{N-1}(x, t=T)=\psi_{f}(x)$, respectively, as input to the gradient ascent optimization in the NIC-CAGE software package.

\subsection{Time Evolution}

With the time-independent Schrödinger equation properly discretized in Section 3.1, we can now proceed with the general case for a time-dependent Hamiltonian and its discretized form. By combining explicit and implicit Euler schemes as in Ref. [32, one obtains the following Crank-Nicolson scheme for a time-dependent Hamiltonian:

$$
\left[1+\frac{i \tau}{2} \mathcal{H}\left(x, t+\frac{\tau}{2}\right)\right] \psi(x, t+\tau)=\left[1-\frac{i \tau}{2} \mathcal{H}\left(x, t+\frac{\tau}{2}\right)\right] \psi(x, t),
$$

where $\tau=\frac{T}{N-1}$ is the time step across a grid of $N$ equidistant points on the interval $[0, T]$. It is important to note that the Crank-Nicolson scheme in 
Eq. (11) is unitary and, therefore, preserves the norm of the wavefunction. We are now in a position to discretize time in a similar manner as we have previously discretized space in Section 3.1. Using a similar notation scheme as before gives $\psi\left(x_{i}, t_{j}\right)=\psi\left(x_{\min }+i \Delta x, j \tau\right)=\psi_{j}^{i}$ where $i=0, \ldots, L-1$ and $j=0, \ldots, N-1$. With this simplified notation, in conjunction with the matrix notation of Eq. (10), the Crank-Nicolson expression in Eq. (11) can be written as the following matrix equation:

$$
\begin{gathered}
\left(\mathbb{I}+\frac{i \tau}{2}\left[-\frac{1}{24 m(\Delta x)^{2}}\left(-\mathbb{I}^{(2-)}+16 \mathbb{I}^{(1-)}-30 \mathbb{I}+16 \mathbb{I}^{(1+)}-\mathbb{I}^{(2+)}\right)+\mathbf{V}_{j+1 / 2}\right]\right) \boldsymbol{\psi}_{j+1}= \\
\left(\mathbb{I}-\frac{i \tau}{2}\left[-\frac{1}{24 m(\Delta x)^{2}}\left(-\mathbb{I}^{(2-)}+16 \mathbb{I}^{(1-)}-30 \mathbb{I}+16 \mathbb{I}^{(1+)}-\mathbb{I}^{(2+)}\right)+\mathbf{V}_{j+1 / 2}\right]\right) \boldsymbol{\psi}_{j},
\end{gathered}
$$

where $\boldsymbol{\psi}_{j}$ is vectorized in space $(\mathbf{x})$ and evaluated at time $t_{j}=j \tau$, where $j=$ $0, \ldots, N-1$ (i.e., $\boldsymbol{\psi}_{j}$ is a column vector with entries $\psi_{j}^{i}$ ). In addition, $\mathbf{V}_{j+1 / 2}$ is a diagonal matrix (cf. Eq. (10) ) evaluated at time $t_{j+1 / 2}=(j+1 / 2) \tau$, where $j=0, \ldots, N-2$. It should also be noted that Eq. (12), by definition, is only valid for values of $j=0, \ldots, N-2$, and $\boldsymbol{\psi}_{0}$, therefore, represents the initial state.

As will be shown in Section 3.3, it is helpful to write Eq. (12) in a more compact notation. To this end, we denote the operator in parentheses on the left-hand side of Eq. (12) as the $L \times L$ matrix $\mathbf{U}_{j+1 / 2}$. Similarly, we represent the operator on the right-hand side of Eq. (12) by the $L \times L$ matrix $\mathbf{W}_{j+1 / 2}$, which results in the following matrix equation:

$$
\mathbf{U}_{j+1 / 2} \boldsymbol{\psi}_{j+1}=\mathbf{W}_{j+1 / 2} \boldsymbol{\psi}_{j}
$$

where $\mathbf{U}_{j+1 / 2}$ and $\mathbf{W}_{j+1 / 2}$ are banded pentadiagonal $L \times L$ matrices of the form

$$
\left(\begin{array}{ccccccc}
a_{1} & b_{1} & d_{1} & 0 & \cdots & 0 \\
c_{2} & a_{2} & b_{2} & d_{2} & & & \vdots \\
e_{3} & c_{3} & a_{3} & b_{3} & d_{3} & & \\
0 & & & \ddots & & & 0 \\
\vdots & & e_{L-2} & c_{L-2} & a_{L-2} & b_{L-2} & d_{L-2} \\
0 & \ldots & & e_{L-1} & c_{L-1} & a_{L-1} & b_{L-1} \\
& & & & e_{L} & c_{L} & a_{L}
\end{array}\right)
$$


Upon closer examination of Eq. (12), one finds that the upper and lower diagonals of Eq. (14) are all constants, while the diagonal entries are functions of the time-dependent potential $V\left(x_{i}, t_{j+1 / 2}\right)=V\left(x_{i}\right)-\mu\left(x_{i}\right) \epsilon\left(t_{j+1 / 2}\right)$, as summarized below:

$$
\begin{gathered}
{\left[\mathbf{U}_{j+1 / 2}\right]_{k l}= \begin{cases}1+\frac{5 i \tau}{8 m(\Delta x)^{2}}+\frac{i \tau}{2}\left[V\left(x_{k}\right)-\mu\left(x_{k}\right) \epsilon\left(t_{j+1 / 2}\right)\right], & l=k \\
-\frac{i \tau}{3 m(\Delta x)^{2}}, & l=k \pm 1 \\
\frac{i \tau}{48 m(\Delta x)^{2}}, & l=k \pm 2\end{cases} } \\
{\left[\mathbf{W}_{j+1 / 2}\right]_{k l}= \begin{cases}1-\frac{5 i \tau}{8 m(\Delta x)^{2}}-\frac{i \tau}{2}\left[V\left(x_{k}\right)-\mu\left(x_{k}\right) \epsilon\left(t_{j+1 / 2}\right)\right], & l=k \\
\frac{i \tau}{3 m(\Delta x)^{2}}, & l=k \pm 1 \\
-\frac{i \tau}{48 m(\Delta x)^{2}}, & l=k \pm 2\end{cases} }
\end{gathered}
$$

Since the matrix-vector product on the right-hand side of Eq. 13 is computed several times for each time step, significant computational savings are utilized in the NIC-CAGE software package by replacing the matrix-vector product with a single pre-computed vector with the following elements:

$$
\begin{array}{r}
r_{j}^{i}=\psi_{j}^{i}-\frac{i \tau}{2}\left[-\frac{1}{24 m(\Delta x)^{2}}\left(-\psi_{j}^{i-2}+16 \psi_{j}^{i-1}-30 \psi_{j}^{i}+16 \psi_{j}^{i+1}-\psi_{j}^{i+2}\right)\right. \\
\left.+V_{j+1 / 2}^{i} \psi_{j}^{i}\right]
\end{array}
$$

where $V_{j+1 / 2}^{i}=V\left(x_{i}, t_{j+1 / 2}\right)=V\left(x_{i}\right)-\mu\left(x_{i}\right) \epsilon\left(t_{j+1 / 2}\right)$.

\subsection{Numerical Optimization}

The numerical procedure presented in the previous section gives the timeevolution of the wavefunction for a given $\epsilon_{j+1 / 2}$ where $j=0, \ldots, N-2$. In this section, we derive analytic gradients and present a numerical maximization method (using a gradient ascent algorithm) that back-propagates the gradient of the objective functional, $J\left[\psi_{N-1}(x), \epsilon\right]$ :

$$
\frac{d J\left[\psi_{N-1}(x), \epsilon\right]}{d \epsilon_{j+1 / 2}}=\frac{d P\left[\psi_{N-1}(x)\right]}{d \epsilon_{j+1 / 2}}+\frac{d F[\epsilon]}{d \epsilon_{j+1 / 2}},
$$

where we have used the definition of $J\left[\psi_{N-1}(x), \epsilon\right]$ from Eq. (6). To make our discussion of this numerical optimization more concrete (and to remind 
the reader of the specific time steps used in this calculation), Fig. 2 shows a schematic of how the electric field optimization process can be conceptualized. As mentioned in Section 3.2 , the time interval $[0, T]$ is discretized across a grid of $N$ equidistant points (endpoints inclusive); however, the electric field amplitudes $\epsilon_{j+1 / 2}$ are only defined at times $t_{j+1 / 2}=(j+1 / 2) \tau$, where $j=0, \ldots, N-2$. Within the small duration $\tau=\frac{T}{N-1}$, the electric field amplitudes, $\epsilon_{j+1 / 2}$, are approximated to be constant. The vertical arrows in Fig. 2 represent the gradients $\frac{d J}{d \epsilon_{j+1 / 2}}$ that indicate how each amplitude $\epsilon_{j+1 / 2}$ should be modified in the next iteration to maximize the objective functional $J$.

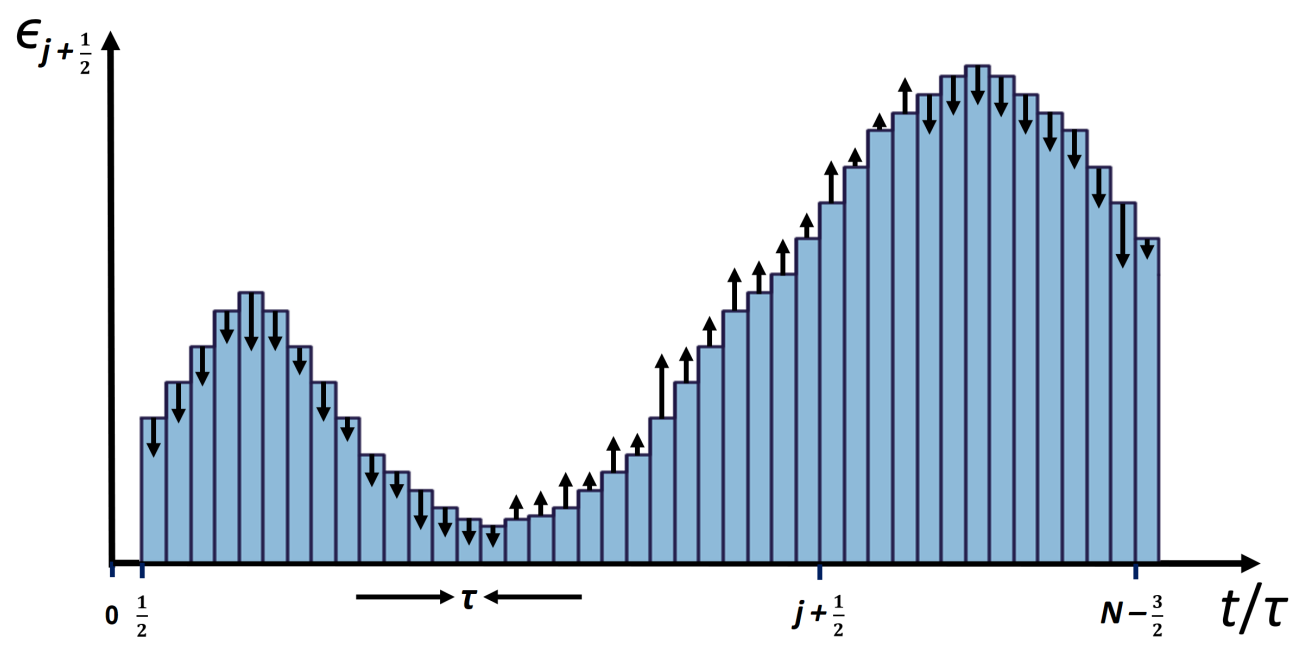

Figure 2: Schematic representation of the electric field amplitudes, $\epsilon_{j+1 / 2}$, defined from time $t=\frac{1}{2} \tau$ to $\left(N-\frac{3}{2}\right) \tau$. During each small interval, the electric field amplitude $\epsilon_{j+1 / 2}$ is approximated to be constant. The vertical arrows represent the gradients $\frac{d J}{d \epsilon_{j+1 / 2}}$ that indicate how each amplitude $\epsilon_{j+1 / 2}$ should be modified in the next iteration to maximize the objective functional $J$.

To enable this numerical optimization across a grid of points, we first approximate the transition probability in Eq. (6) as

$$
\begin{aligned}
P\left[\psi_{N-1}(x)\right] & =\left|\int_{-\infty}^{\infty} \psi_{f}^{*}(x) \psi_{N-1}(x) d x\right|^{2} \\
& \approx\left|\boldsymbol{\psi}_{\boldsymbol{f}}^{\dagger} \cdot \boldsymbol{\psi}_{\boldsymbol{N - 1}}\right|^{2}(\Delta x)^{2}
\end{aligned}
$$

where $\boldsymbol{\psi}_{\boldsymbol{f}}$ is a known final target wavefunction (given by the user, as described in Section 2), and $\boldsymbol{\psi}_{N-\mathbf{1}}$ is obtained after applying $N-1$ iterations of Eq. 
(13). For clarification, Eq. (19) uses the same notation as Section 3.2, where $\boldsymbol{\psi}_{N-1}$ is a column vector with entries $\psi_{N-1}^{i}$ and $\boldsymbol{\psi}_{\boldsymbol{f}}^{\dagger}$ is the complex conjugate transpose of $\boldsymbol{\psi}_{\boldsymbol{f}}$ (i.e., $\boldsymbol{\psi}_{\boldsymbol{f}}^{\dagger}$ is a row vector with entries $\left.\left(\psi_{f}^{i}\right)^{*}\right)$. With these definitions, it follows that $P \leq 1$.

Similarly, the fluence in Eq. (7) can be approximated as

$$
\begin{aligned}
F[\epsilon] & =-\alpha \int_{0}^{T} \epsilon(t)^{2} d t \\
& \approx-\alpha \tau \boldsymbol{\epsilon} \cdot \boldsymbol{\epsilon},
\end{aligned}
$$

To evaluate Eq. (18), we commence by first obtaining expressions for the derivatives of $P$ with respect to each $\epsilon_{j+1 / 2}$ value using the chain rule of Wirtinger's derivatives [33].

$$
\begin{aligned}
\frac{d P}{d \epsilon_{j+1 / 2}} & =\left(\frac{\partial\left|\boldsymbol{\psi}_{\boldsymbol{f}}^{\dagger} \cdot \boldsymbol{\psi}_{\boldsymbol{N}-\mathbf{1}}\right|^{2}}{\partial\left(\boldsymbol{\psi}_{\boldsymbol{f}}^{\dagger} \cdot \boldsymbol{\psi}_{\boldsymbol{N - 1}}\right)} \frac{d\left(\boldsymbol{\psi}_{\boldsymbol{f}}^{\dagger} \cdot \boldsymbol{\psi}_{\boldsymbol{N}-\mathbf{1}}\right)}{d \epsilon_{j+1 / 2}}+\frac{\partial\left|\boldsymbol{\psi}_{\boldsymbol{f}}^{\dagger} \cdot \boldsymbol{\psi}_{\boldsymbol{N - 1}}\right|^{2}}{\partial\left(\boldsymbol{\psi}_{\boldsymbol{f}}^{\dagger} \cdot \boldsymbol{\psi}_{\boldsymbol{N - 1}}\right)^{*}} \frac{d\left(\boldsymbol{\psi}_{\boldsymbol{f}}^{\dagger} \cdot \boldsymbol{\psi}_{\boldsymbol{N}-\mathbf{1}}\right)^{*}}{d \epsilon_{j+1 / 2}}\right)(\Delta x)^{2} \\
& =2 \times \Re\left\{\left(\boldsymbol{\psi}_{\boldsymbol{f}}^{\dagger} \cdot \boldsymbol{\psi}_{\boldsymbol{N}-\mathbf{1}}\right) \frac{d\left(\boldsymbol{\psi}_{\boldsymbol{f}}^{\dagger} \cdot \boldsymbol{\psi}_{\boldsymbol{N}-\mathbf{1}}\right)^{*}}{d \epsilon_{j+1 / 2}}\right\}(\Delta x)^{2},
\end{aligned}
$$

where

$$
\begin{aligned}
\frac{d\left(\boldsymbol{\psi}_{\boldsymbol{f}}^{\dagger} \cdot \boldsymbol{\psi}_{\boldsymbol{N - 1}}\right)}{d \epsilon_{j+1 / 2}} & =\frac{d\left(\boldsymbol{\psi}_{\boldsymbol{f}}^{\dagger} \cdot \boldsymbol{\psi}_{\boldsymbol{N}-\mathbf{1}}\right)}{d \boldsymbol{\psi}_{\boldsymbol{N}-\mathbf{1}}} \frac{d \boldsymbol{\psi}_{\boldsymbol{N - 1}}}{d \epsilon_{j+1 / 2}} \\
& =\boldsymbol{\psi}_{\boldsymbol{f}}^{\dagger} \cdot \frac{d \boldsymbol{\psi}_{\boldsymbol{N - 1}}}{d \epsilon_{j+1 / 2}}
\end{aligned}
$$

As such, our gradient ascent algorithm culminates in finding an expression for $\frac{d \psi_{N-1}}{d \epsilon_{j+1 / 2}}$. To obtain a numerically tractable expression for this derivative, we note that $\boldsymbol{\psi}_{j+1}$ can be propagated from $\boldsymbol{\psi}_{j}$ by inverting Eq. (13); i.e.,

$$
\boldsymbol{\psi}_{j+1}=\mathbf{U}_{j+1 / 2}^{-1} \mathbf{W}_{j+1 / 2} \boldsymbol{\psi}_{j}
$$

where we recall that the above expression, by definition, is only valid for values of $j=0, \ldots, N-2$ (cf. Eq. (12)). It follows that repeated iteration of Eq. (23) gives

$$
\begin{array}{r}
\boldsymbol{\psi}_{N-1}=\left(\mathbf{U}_{N-3 / 2}^{-1} \mathbf{W}_{N-3 / 2}\right)\left(\mathbf{U}_{N-5 / 2}^{-1} \mathbf{W}_{N-5 / 2}\right) \\
\ldots\left(\mathbf{U}_{j+1 / 2}^{-1} \mathbf{W}_{j+1 / 2}\right) \cdots\left(\mathbf{U}_{3 / 2}^{-1} \mathbf{W}_{3 / 2}\right)\left(\mathbf{U}_{1 / 2}^{-1} \mathbf{W}_{1 / 2}\right) \boldsymbol{\psi}_{0} .
\end{array}
$$


We have specifically chosen to write Eq. 23 in this suggestive form since only the diagonal entries of $\mathbf{U}_{j+1 / 2}$ and $\mathbf{W}_{j+1 / 2}$ are a function of $\epsilon_{j+1 / 2}$ (cf., Eqs. (13), (15), and (16)). Therefore:

$$
\begin{aligned}
& \frac{d \boldsymbol{\psi}_{N-1}}{d \epsilon_{j+1 / 2}}=\left(\mathbf{U}_{N-3 / 2}^{-1} \mathbf{W}_{N-3 / 2}\right)\left(\mathbf{U}_{N-5 / 2}^{-1} \mathbf{W}_{N-5 / 2}\right) \\
\cdots & {\left[\left(\frac{d \mathbf{U}_{j+1 / 2}^{-1}}{d \epsilon_{j+1 / 2}}\right)\left(\mathbf{W}_{j+1 / 2}\right)+\left(\mathbf{U}_{j+1 / 2}^{-1}\right)\left(\frac{d \mathbf{W}_{j+1 / 2}}{d \epsilon_{j+1 / 2}}\right)\right] \cdots\left(\mathbf{U}_{3 / 2}^{-1} \mathbf{W}_{3 / 2}\right)\left(\mathbf{U}_{1 / 2}^{-1} \mathbf{W}_{1 / 2}\right) \boldsymbol{\psi}_{0} } \\
= & \left(\prod_{k=j+1}^{N-2} \mathbf{U}_{k+1 / 2}^{-1} \mathbf{W}_{k+1 / 2}\right)\left[\left(\frac{d \mathbf{U}_{j+1 / 2}^{-1}}{d \epsilon_{j+1 / 2}}\right)\left(\mathbf{W}_{j+1 / 2}\right)+\left(\mathbf{U}_{j+1 / 2}^{-1}\right) \operatorname{diag}\left(\frac{i \tau}{2} \boldsymbol{\mu}\right)\right] \boldsymbol{\psi}_{j} .
\end{aligned}
$$

where $\operatorname{diag}\left(\frac{i \tau}{2} \boldsymbol{\mu}\right)$ denotes a diagonal matrix having the entries $\frac{i \tau}{2} \boldsymbol{\mu}$ along the diagonal, and $\prod_{k=j+1}^{N-2} \mathbf{U}_{k+1 / 2}^{-1} \mathbf{W}_{k+1 / 2}$ denotes a "time-ordered product," where matrices at earlier times are to the right of matrices at later times. Eq. 25) can be simplified even further by using the following identity for the derivative of a matrix inverse: $\frac{d \mathbf{A}^{-1}}{d x}=-\mathbf{A}^{-1} \frac{d \mathbf{A}}{d x} \mathbf{A}^{-1}$. Therefore,

$$
\begin{gathered}
\frac{d \boldsymbol{\psi}_{N-\mathbf{1}}}{d \epsilon_{j+1 / 2}}=\left(\prod_{k=j+1}^{N-2} \mathbf{U}_{k+1 / 2}^{-1} \mathbf{W}_{k+1 / 2}\right)\left[\left(-\mathbf{U}_{j+1 / 2}^{-1} \frac{d \mathbf{U}_{j+1 / 2}}{d \epsilon_{j+1 / 2}} \mathbf{U}_{j+1 / 2}^{-1}\right)\left(\mathbf{W}_{j+1 / 2}\right)\right. \\
\left.+\left(\mathbf{U}_{j+1 / 2}^{-1}\right) \operatorname{diag}\left(\frac{i \tau}{2} \boldsymbol{\mu}\right)\right] \boldsymbol{\psi}_{j} \\
=\left(\prod_{k=j+1}^{N-2} \mathbf{U}_{k+1 / 2}^{-1} \mathbf{W}_{k+1 / 2}\right)\left[-\mathbf{U}_{j+1 / 2}^{-1} \operatorname{diag}\left(-\frac{i \tau}{2} \boldsymbol{\mu}\right) \mathbf{U}_{j+1 / 2}^{-1} \mathbf{W}_{j+1 / 2}\right. \\
\left.=\left(\prod_{k=j+1}^{N-2} \mathbf{U}_{k+1 / 2}^{-1} \mathbf{W}_{k+1 / 2}^{-1}\right)\left[-\mathbf{U}_{j+1 / 2}^{-1} \operatorname{diag}\left(\frac{i \tau}{2} \boldsymbol{\mu}\right)\right] \boldsymbol{\psi}_{j} \operatorname{diag}\left(-\frac{i \tau}{2} \boldsymbol{\mu}\right) \boldsymbol{\psi}_{j+1}+\mathbf{U}_{j+1 / 2}^{-1} \operatorname{diag}\left(\frac{i \tau}{2} \boldsymbol{\mu}\right) \boldsymbol{\psi}_{j}\right] \\
=\frac{i \tau}{2}\left(\prod_{k=j+1}^{N-2} \mathbf{U}_{k+1 / 2}^{-1} \mathbf{W}_{k+1 / 2}\right) \mathbf{U}_{j+1 / 2}^{-1}\left[\boldsymbol{\mu} \odot\left(\boldsymbol{\psi}_{j+1}+\boldsymbol{\psi}_{j}\right)\right]
\end{gathered}
$$


where the $\odot$ symbol denotes the Schur (element-wise) product (i.e., $\boldsymbol{\mu} \odot$ $\left(\boldsymbol{\psi}_{j+1}+\boldsymbol{\psi}_{j}\right)$ is a column vector with entries $\left.\mu^{i}\left(\psi_{j+1}^{i}+\psi_{j}^{i}\right)\right)$. It should also be mentioned that $\frac{d \psi_{N-1}}{d \epsilon_{j+1 / 2}}$, by definition, is only defined for values of $j=$ $0, \ldots, N-2$ (see Eqs. (12), (23), and recall that the "latest" electric field that $\boldsymbol{\psi}_{N-1}$ can depend on is the previous $\epsilon_{N-3 / 2}$, not the future $\left.\epsilon_{N-1 / 2}\right)$. However, it should be pointed out that Eq. (26) cannot be directly evaluated for $j=N-2$ since the lower index of the product symbol then becomes larger than the upper index. To evaluate $\frac{d \psi_{N-1}}{d \epsilon_{j+1 / 2}}$ for the special case when $j=N-2$, we can use the original definition of $\boldsymbol{\psi}_{N-1}$ to obtain

$$
\begin{aligned}
\frac{d \boldsymbol{\psi}_{N-1}}{d \epsilon_{N-3 / 2}}= & {\left[\frac{d}{d \epsilon_{N-3 / 2}}\left(\mathbf{U}_{N-3 / 2}^{-1} \mathbf{W}_{N-3 / 2}\right)\right]\left(\mathbf{U}_{N-5 / 2}^{-1} \mathbf{W}_{N-5 / 2}\right) } \\
& \cdots\left(\mathbf{U}_{j+1 / 2}^{-1} \mathbf{W}_{j+1 / 2}\right) \cdots\left(\mathbf{U}_{3 / 2}^{-1} \mathbf{W}_{3 / 2}\right)\left(\mathbf{U}_{1 / 2}^{-1} \mathbf{W}_{1 / 2}\right) \boldsymbol{\psi}_{0} \\
& =\left[\frac{d}{d \epsilon_{N-3 / 2}}\left(\mathbf{U}_{N-3 / 2}^{-1} \mathbf{W}_{N-3 / 2}\right)\right] \boldsymbol{\psi}_{N-2} .
\end{aligned}
$$

Using the same mathematical techniques and identities as in Eqs. 225 - (26) finally gives

$$
\frac{d \boldsymbol{\psi}_{N-\mathbf{1}}}{d \epsilon_{N-3 / 2}}=\frac{i \tau}{2} \mathbf{U}_{N-3 / 2}^{-1} \boldsymbol{\mu} \odot\left[\left(\mathbf{U}_{N-3 / 2}^{-1} \mathbf{W}_{N-3 / 2}+\mathbb{I}\right) \boldsymbol{\psi}_{N-2}\right] .
$$

For convenience, we summarize the closed form expressions for $\frac{d \psi_{N-1}}{d \epsilon_{j+1 / 2}}$ for all possible values of $j$ :

$$
\frac{d \boldsymbol{\psi}_{N-\mathbf{1}}}{d \epsilon_{j+1 / 2}}= \begin{cases}\frac{i \tau}{2} \mathbf{U}_{N-3 / 2}^{-1}\left[\boldsymbol{\mu} \odot\left(\boldsymbol{\psi}_{N-1}+\boldsymbol{\psi}_{N-2}\right)\right], & j=N-2 \\ \frac{i \tau}{2} \mathbf{U}_{N-3 / 2}^{-1} \mathbf{W}_{N-3 / 2} \mathbf{U}_{N-5 / 2}^{-1}\left[\boldsymbol{\mu} \odot\left(\boldsymbol{\psi}_{N-2}+\boldsymbol{\psi}_{N-3}\right)\right], & j=N-3 \\ \frac{i \tau}{2}\left(\prod_{k=j+1}^{N-2} \mathbf{U}_{k+1 / 2}^{-1} \mathbf{W}_{k+1 / 2}\right) \mathbf{U}_{j+1 / 2}^{-1}\left[\boldsymbol{\mu} \odot\left(\boldsymbol{\psi}_{j+1}+\boldsymbol{\psi}_{j}\right)\right], & 0 \leq j \leq N-4\end{cases}
$$

Now that we have a closed form expression for $\frac{d \psi_{N-1}}{d \epsilon_{j+1 / 2}}$, the only remaining quantity needed to evaluate the gradient of the objective functional (i.e., $\frac{d J\left[\psi_{N-1}(x), \epsilon\right]}{d \epsilon_{j+1 / 2}}$ in Eq. (18)) is the derivative of the fluence term (Eq. (20)), which fortunately has a relatively simple form:

$$
\frac{d F[\epsilon]}{d \epsilon_{j+1 / 2}}=-2 \alpha \tau \epsilon_{j+1 / 2}
$$


With these results, we can now numerically solve for the optimized, timedependent, external electric field at each $l^{\text {th }}$ iteration step, $\epsilon_{j+1 / 2}^{(l)}$, to give

$$
\begin{aligned}
\epsilon_{j+1 / 2}^{(l)} & =\epsilon_{j+1 / 2}^{(l-1)}+\gamma \frac{d J\left[\psi_{N-1}(x), \epsilon\right]}{d \epsilon_{j+1 / 2}} \\
& =\epsilon_{j+1 / 2}^{(l-1)}+\gamma\left(\frac{d P\left[\psi_{N-1}(x)\right]}{d \epsilon_{j+1 / 2}}+\frac{d F[\epsilon]}{d \epsilon_{j+1 / 2}}\right) \\
& =\epsilon_{j+1 / 2}^{(l-1)}+\gamma\left(2 \times \Re\left\{\left(\boldsymbol{\psi}_{f}^{\dagger} \cdot \boldsymbol{\psi}_{N-1}\right)\left(\boldsymbol{\psi}_{f}^{\dagger} \cdot \frac{d \boldsymbol{\psi}_{N-1}}{d \epsilon_{j+1 / 2}}\right)^{*}\right\}(\Delta x)^{2}-2 \alpha \tau \epsilon_{j+1 / 2}\right),
\end{aligned}
$$

where we have made use of Eqs. (18), 21), 222), and (30) in the above expression. Finally, $\frac{d \psi_{N-1}}{d \epsilon_{j+1 / 2}}$ can be calculated using Eq. 290 , and $\gamma$ in Eq. (31) is the learning rate of the gradient ascent algorithm, which we calculate using the bisection line-search approach [34] described in Ref. [35]. In short, the gradient ascent algorithm proceeds until the transition probability defined in Eq. (19) reaches a specified tolerance or exceeds a maximum number of iterations (set to 0.99 and 100, respectively, in the NIC-CAGE code). We conclude this section with the following algorithm flowchart that summarizes the various inputs and algorithmic steps used in the NIC-CAGE software package to calculate the optimized electric field $\epsilon(t)$ : 


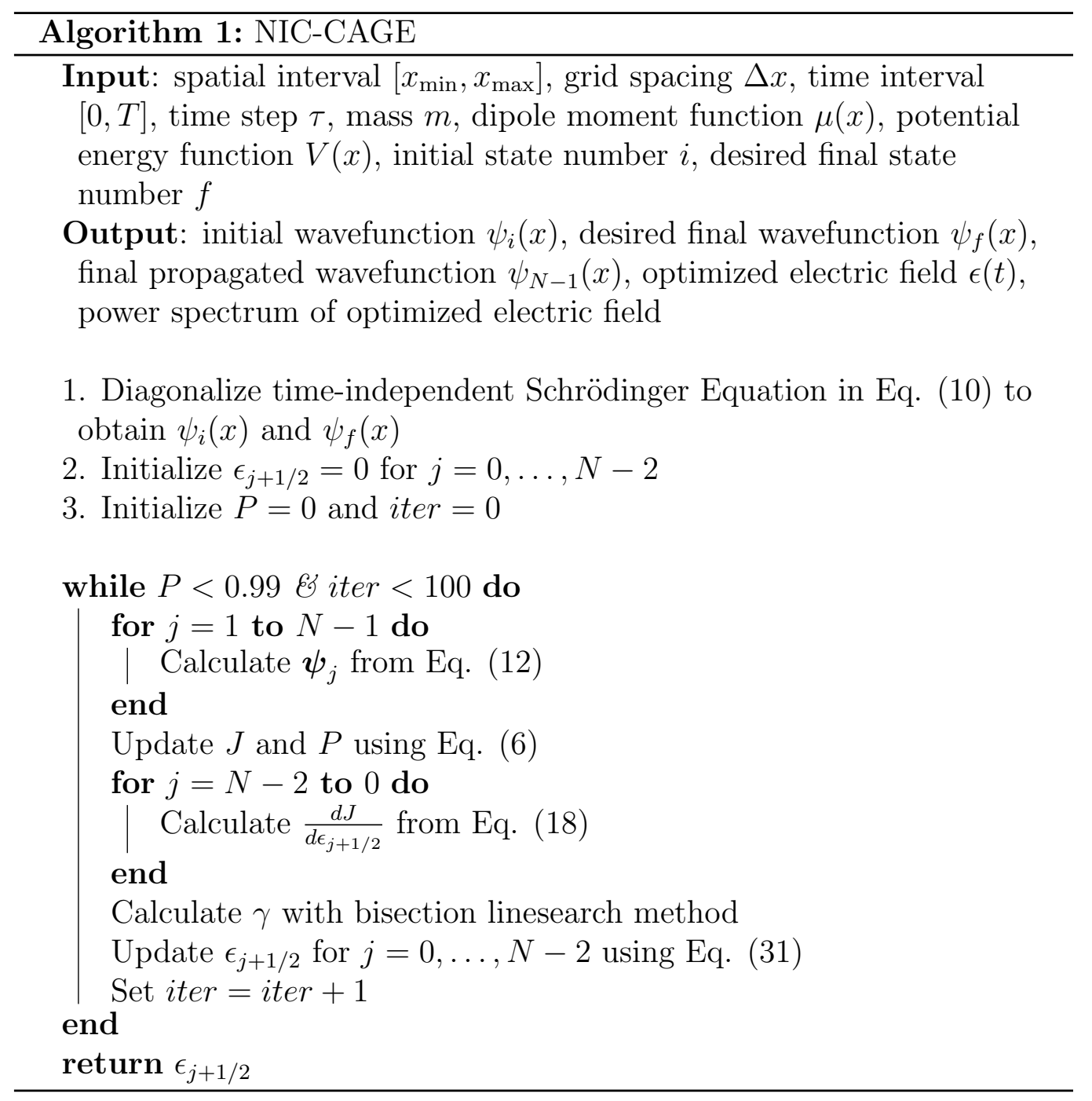

It is worth noting that our implementation can be scaled straightforwardly to higher dimensions by vectorizing the spatial grid in the other dimensions, which still results in a system of linear equations in matrix form [36]. Most notably, the gradient formulation developed in this work remains unchanged for higher dimensional systems since the control field, $\epsilon_{j}$, continues to appear in the diagonal entries only. 


\section{Numerical Examples and Results}

As mentioned in Section 2, the time-independent portions of Eq. (4) [i.e., $V(x)$ and $\mu(x)$ ] can be obtained from a quantum chemistry calculation or take any arbitrary form. To demonstrate the flexibility and capabilities of the NIC-CAGE software package, we give a few representative results of running the NIC-CAGE code on two example potentials that mimic a bond stretching/dissociation process in a photocatalytic reaction. All of the parameters for both potentials (expressed in atomic units) are included in the easy-to-use demo1.m, demo2.m, and demo3.m scripts.

\subsection{Morse Potential}

For our first example, we perform a quantum control optimization using a Morse potential that mimics the photo-induced stretching of an $\mathrm{O}-\mathrm{H}$ bond [37]. The spatial dependence, $V(x)$, of the Morse potential that we used in Eq. (4) has the following form:

$$
V(x)=D_{e}\left(e^{-\beta\left(x-x_{0}\right)}-1\right)^{2}-D_{e},
$$

where the well depth, width, and equilibrium bond distance were taken from Ref. [37] and are given by $D_{e}=0.1994, \beta=1.189$, and $x_{0}=1.821$, respectively. In addition, the dipole moment function that we used in Eq. (4) has the functional form

$$
\mu(x)=\mu_{0} \cdot x \cdot e^{-\frac{x}{x^{*}}},
$$

where $\mu_{0}=3.088$ and $x^{*}=0.6$. The value of $m$ that we used in Eq. (2) is the reduced mass of oxygen and hydrogen. A snippet from the included demo1.m MATLAB script given below provides values of the parameters used in this section:

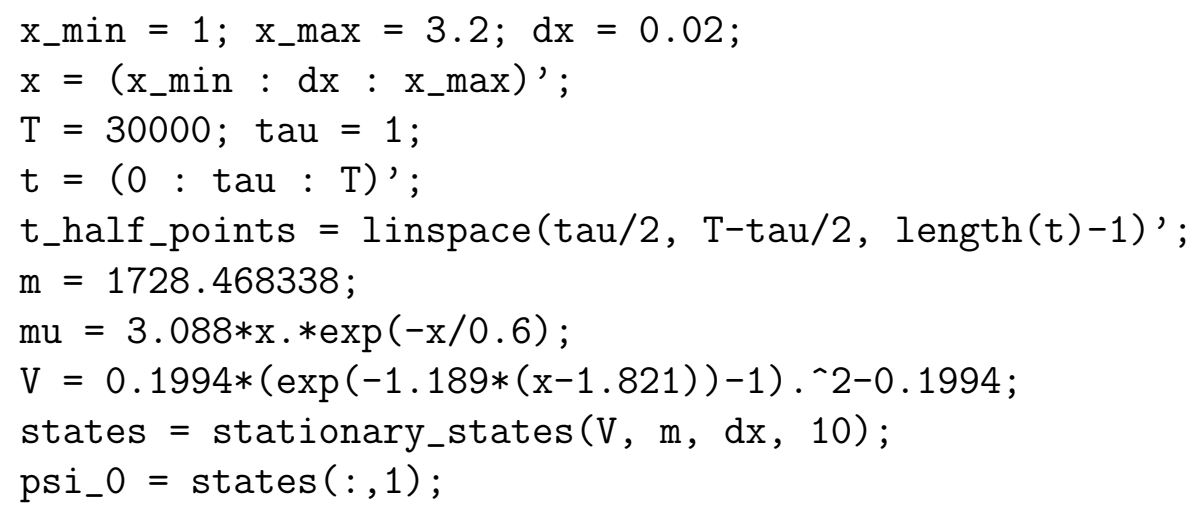


psi_f $=\operatorname{states}(:, 2)$;

Ef $=$ zeros(length(t_half_points), 1);

alpha $=1$;

These values correspond to the variables described previously in this paper as follows:

- $\mathbf{x}_{-} \min , \mathrm{x}_{\text {max }}$, dx: spatial range specified by $\left[\mathbf{x}_{\min }, \mathbf{x}_{\max }\right]$, grid spacing $\Delta \mathrm{x}$

- T, tau: propagation time $T$, time step $\tau$

- $\mathrm{m}$ : effective mass $m$ associated with the molecular motion along the reaction path

- mu: dipole moment function $\mu(x)$, cf. Eq. (33)

- V: Morse potential $V(x)$, cf. Eq. (32)

- psi_0: initial state $\psi_{0}(x)$

- psi_f: final state $\psi_{f}(x)$

- Ef: electric field $\epsilon(t)$

- alpha: fluence penalty pre-factor $\alpha$, cf. Eq. (7)

The quantum control optimization commences with an initial guess of $\epsilon(t)=0$ with $\alpha=1$. It is worth mentioning that this specific choice of initial conditions works for all the examples presented in this paper; however, larger energy transitions may require a non-zero initial guess. Throughout this section, we use the notation mentioned at the end of Section 2 where transitions between the initial and final vibrational eigenstates, $\psi_{i}(x)$ and $\psi_{f}(x)$, are simply denoted by $\nu_{i}$ and $\nu_{f}$, respectively, where $\nu=0$ represents the zero-point ground state.

Fig. 3 depicts the values of both the objective functional, $J$, and transition probability, $P$, as a function of the number of iterations (iteration zero corresponds to the initial values of $J$ or $P$ with an initial guess of $\epsilon(t)=0)$. For the transition $\nu_{i}=0 \rightarrow \nu_{f}=1$, only 3 steps are required to reach a transition probability of $99.2 \%$. Since the vertical axis in Fig. 3 is scaled linearly, it would be incorrect to infer that the improvement at iteration number one is minimal. We can fully appreciate the progress made at the first iterative step by computing the percentage change in $J$ from the previous step. For instance, at the first iteration, the value of $J$ increases from $1.03 \times 10^{-27}$ to $6.19 \times 10^{-9}$, which translates to an increase by $6.01 \times 10^{20 \%}$ - the highest among all other iterations. This overall trend is consistent with all the numerical examples discussed in this paper. 


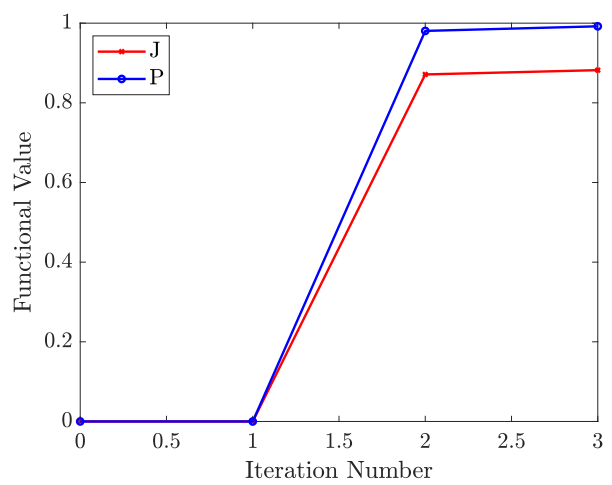

Figure 3: Objective functional, $J$, and transition probability, $P$, as a function of the number of iterations for a quantum control optimization of the $\nu_{i}=0 \rightarrow \nu_{f}=1$ transition in a Morse potential.

Fig. 4 summarizes the various outputs and post-processed results of the NIC-CAGE software package for the $\nu_{i}=0 \rightarrow \nu_{f}=1$ transition in a Morse potential. The panel in Fig. 4a shows the norm-squared vibrational eigenstate, $\left|\psi_{i}(x)\right|^{2}$, with $\nu_{i}=0$ (dashed red line), superimposed on the Morse potential energy function (solid blue line). The optimized electric field in Fig. 4b is nearly a pure sinusoid, which is also reflected in the frequency domain as a sharp peak at around $112 \mathrm{THz}$ in Fig. 4c. It is worth noting that the peak frequency matches the resonant frequency required for the transition between the ground and first excited state. As a check on the fidelity of the results, Fig. 4d shows that the norm-square of the propagated wavefunction, $\left|\psi_{N-1}\right|^{2}$, closely matches the desired target $\left|\psi_{f}\right|^{2}$. As a side note, it should be mentioned that the propagated wavefunction, $\psi_{N-1}$, is complex-valued in general and has both real and imaginary parts (not shown in Fig. 4d); nevertheless, the transition probability $\left|\int_{-\infty}^{\infty} \psi_{f}^{*}(x) \psi_{N-1}(x) d x\right|^{2}$ is real-valued and close to unity, emphasizing the ability of the NIC-CAGE software package to directly optimize the control field $\epsilon(t)$ in this system. 

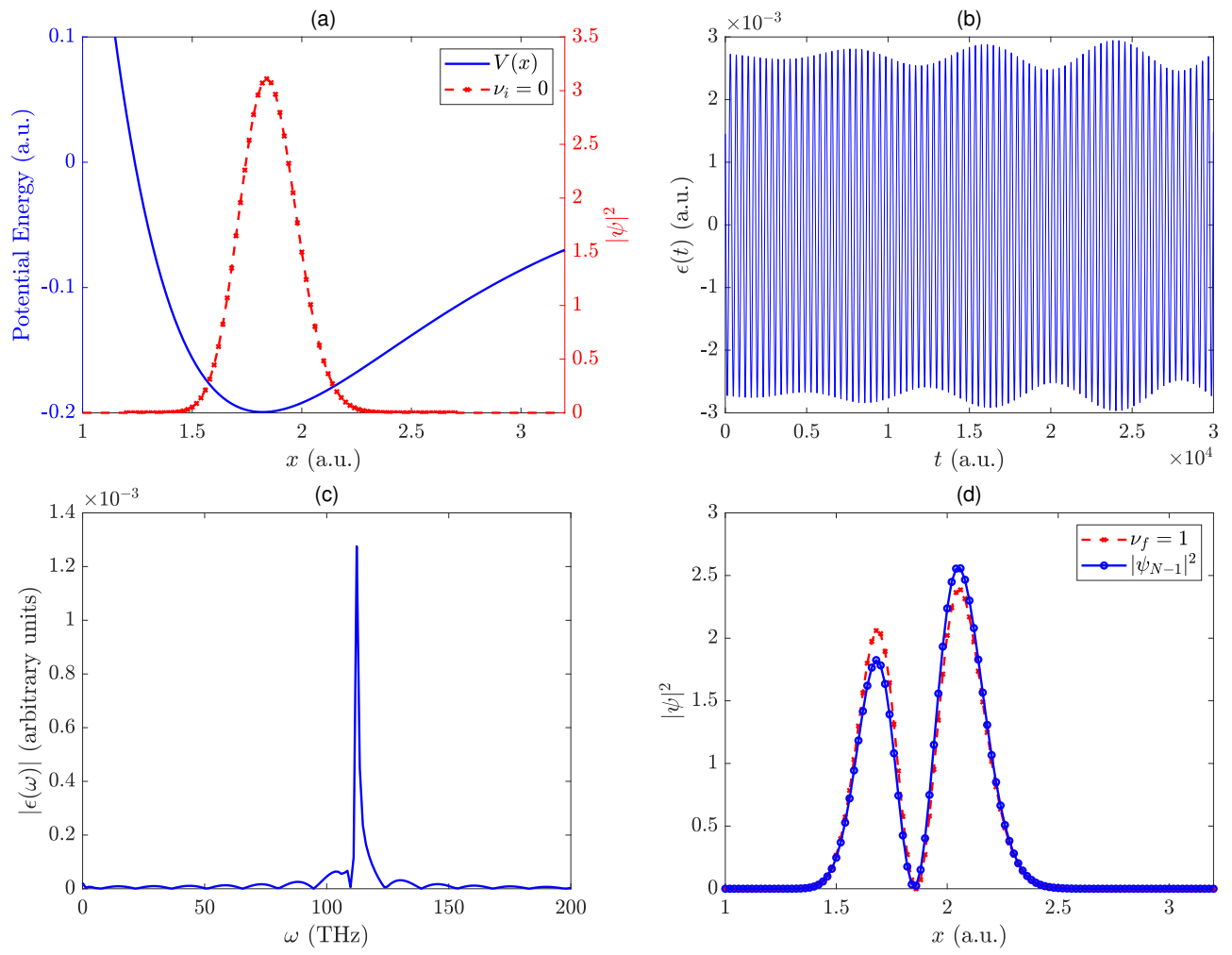

Figure 4: (a) Morse potential energy (solid blue line) and norm-squared initial vibrational eigenstate, $\left|\psi_{i}(x)\right|^{2}$, with $\nu_{i}=0$ (red dashed line). The numerical value of the potential energy and probability density can be measured from the left and right vertical axes, respectively. (b) Optimized electric field as a function of time for the $\nu_{i}=0 \rightarrow \nu_{f}=1$ transition. (c) Power spectrum of the optimized electric field. The dominant frequency is approximately $112 \mathrm{THz}$. (d) Norm-squared final target wavefunction $\left|\psi_{f}(x)\right|^{2}$ with $\nu_{f}=1$ and the propagated wavefunction $\left|\psi_{N-1}\right|^{2}$, which achieves a transition probability of $P=0.992$.

In our next example, we attempt a more difficult case with the NIC-CAGE software package that involves a transition between non-adjacent energy levels (included in the demo2.m MATLAB script). Fig. 5 shows the convergence behavior of both the objective functional, $J$, and transition probability, $P$, as a function of the number of iterations for the $\nu_{i}=3 \rightarrow \nu_{f}=5$ transition. After only two iteration steps, over $88 \%$ of the maximum objective functional value is achieved, and the subsequent steps give an incremental improvement before terminating at the 44th step with $99.1 \%$ probability. It is interesting to note that terminating the optimization prematurely after only two 
iterations results in an electric field that is almost a pure sinusoid at approximately $188 \mathrm{THz}$. As such, the subsequent iterations clearly show that the fluence term (i.e., Eq. (20)) significantly impacts the overall objective functional value with the algorithm effectively penalizing the dominant frequency component, allowing for multi-photon transitions to occur (discussed further below).

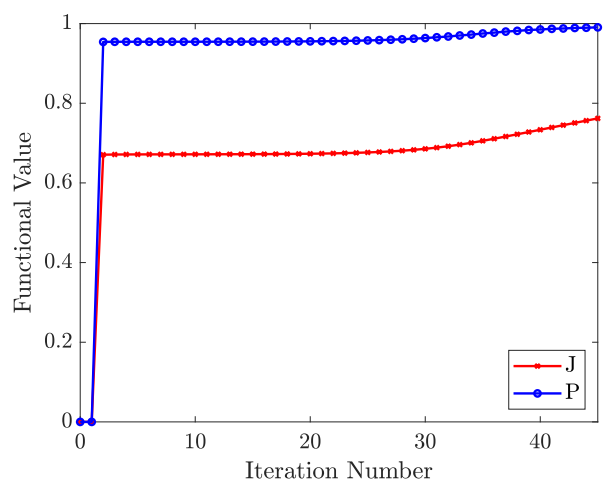

Figure 5: Objective functional, $J$, and transition probability, $P$, as a function of the number of iterations for a quantum control optimization of the $\nu_{i}=3 \rightarrow \nu_{f}=5$ transition in a Morse potential.

Fig. 6 summarizes the various outputs and post-processed results of the NIC-CAGE software package for the $\nu_{i}=3 \rightarrow \nu_{f}=5$ transition in a Morse potential. The panel in Fig. 6a shows the norm-squared initial vibrational eigenstate, $\left|\psi_{i}(x)\right|^{2}$, with $\nu_{i}=3$ (dashed red line), superimposed on the Morse potential energy function (solid blue line). The optimized electric field in Fig. 6b is signficantly more complicated than the $\nu_{i}=0 \rightarrow \nu_{f}=1$ case discussed previously. The power spectrum of the optimized electric field at the end of 44th iteration step, shown in Fig. 6c, now contains three peaks that correspond to the $\nu=3 \rightarrow 4, \nu=4 \rightarrow 5$ and $\nu=3 \rightarrow 5$ transitions. While the single-photon transition has a higher intensity than the peaks corresponding to the $\nu=3 \rightarrow 4 \rightarrow 5$ transition, this example shows that using a single "trivial" frequency corresponding to the energy difference between the ground and excited state may not be sufficient, and a fully numerical optimization as implemented in the NIC-CAGE code is necessary. Finally, as a check on the fidelity of the results, Fig. 6d shows that the normsquare of the propagated wavefunction, $\left|\psi_{N-1}\right|^{2}$, closely matches the desired target $\left|\psi_{f}\right|^{2}$. 

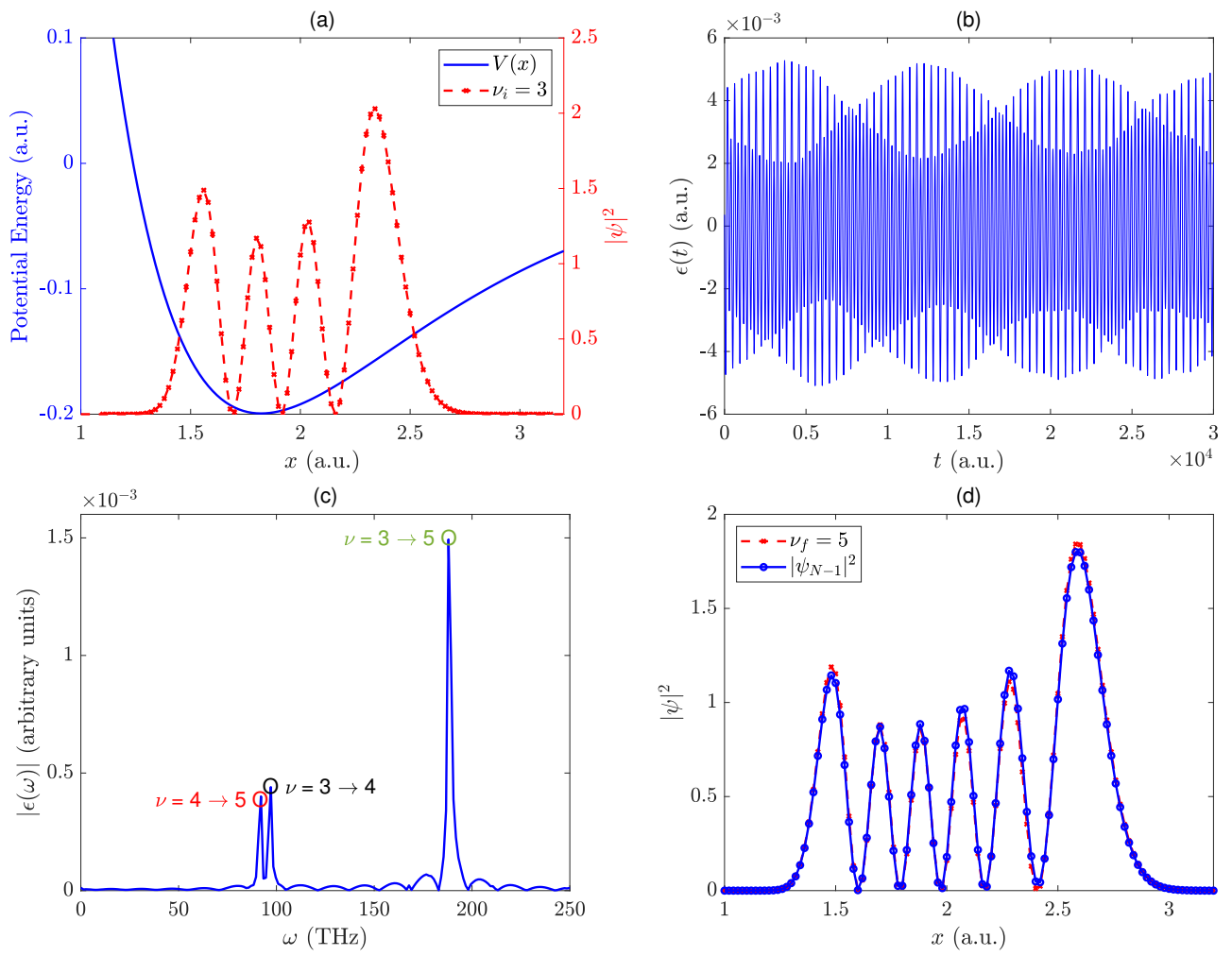

Figure 6: (a) Morse potential energy (solid blue line) and norm-squared initial vibrational eigenstate, $\left|\psi_{i}(x)\right|^{2}$, with $\nu_{i}=3$ (red dashed line). The numerical value of the potential energy and probability density can be measured from the left and right vertical axes, respectively. (b) Optimized electric field as a function of time for the $\nu_{i}=3 \rightarrow \nu_{f}=5$ transition. (c) Power spectrum of the optimized electric field. Three major peaks at approximately 92, 97, and $188 \mathrm{THz}$ can be seen, which correspond to the resonant frequencies in the labelled transitions. (d) Norm-squared final target wavefunction $\left|\psi_{f}(x)\right|^{2}$ with $\nu_{f}=5$ and the propagated wavefunction $\left|\psi_{N-1}\right|^{2}$, which achieves a transition probability of $P=0.991$.

\subsection{Asymmetric Double-Well Potential}

To test the robustness of the NIC-CAGE software package, we now perform a quantum control optimization on a different potential energy function, namely an asymmetric double-well potential:

$$
V(x)=\frac{x^{4}}{64}-\frac{x^{2}}{4}+\frac{x^{3}}{256} .
$$

The functional form of this double-well potential captures all the basic physics of a donor-acceptor reactant/product photochemical system. Specifically, the 
initial ground state with $\nu_{i}=0$ is localized in the deeper "electron donor reactant" left well, and the desired vibrationally-excited final state with $\nu_{f}=1$ is primarily confined to the "electron acceptor product" well on the right (cf. Figs. $8 \mathrm{a}$ and $8 \mathrm{~d}$ ). In addition to examining a completely new potential energy function, this particular example investigates the effects of a significantly shorter pulse on the optimization. For this specific example, we chose a unit mass with a dipole moment given by $\mu(x)=x$. The following code snippet from the included demo3.m MATLAB script shows only those initialization variables that differ from the previous section:

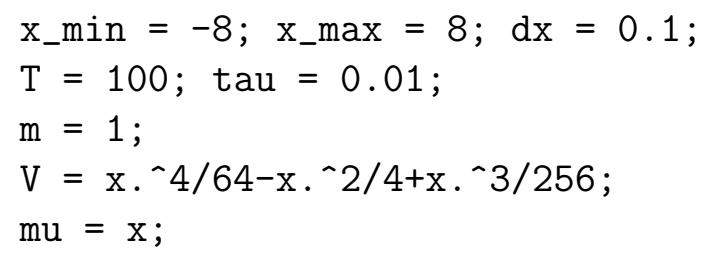

For the $\nu_{i}=0 \rightarrow \nu_{f}=1$ transition in the asymmetric double-well potential, we examined the effect of using two different propagation times: $T=1000$ and 100 a.u. As illustrated in Fig. 7a for $T=1000$, we observed a rather quick convergence to a transition probability of 1.000 within 5 iteration steps. The corresponding optimized electric field for this case, not shown for brevity, is essentially a pure sinusoid at 1,041 THz, the resonant frequency associated with the desired transition. In contrast, for $T=100$, the transition probability reaches 0.975 only after a lengthy 100 iteration steps (cf. Fig. 7b).
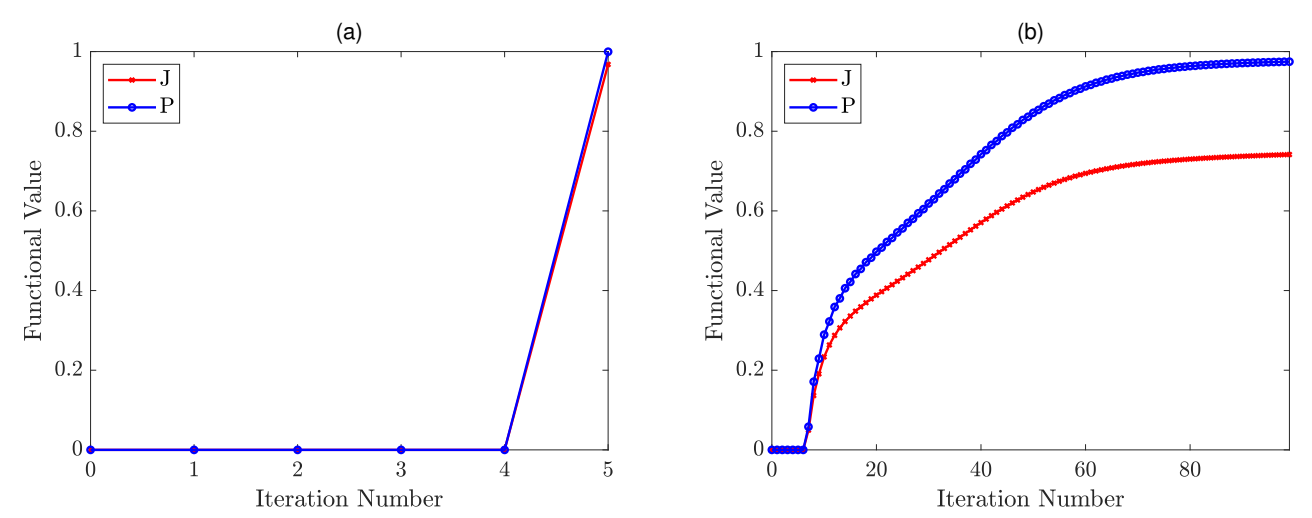

Figure 7: Objective functional, $J$, and transition probability, $P$, as a function of the number of iterations for a quantum control optimization of the $\nu_{i}=0 \rightarrow \nu_{f}=1$ transition in an asymmetric double-well potential for (a) $T=1000$ a.u. and (b) $T=100$ a.u. 
Since the solutions of the asymmetric double-well potential for $T=1000$ are relatively trivial, we only present the various outputs and post-processed results of the NIC-CAGE software package for the $T=100$ case in Fig. 8. The panel in Fig. 8a shows the norm-squared initial vibrational eigenstate, $\left|\psi_{i}(x)\right|^{2}$, with $\nu_{i}=0$ (dashed red line), superimposed on the asymmetric double-well potential energy function (solid blue line). The optimized electric field in Fig. 8b is clearly a superposition of numerous frequencies, which is more complicated than either of the cases discussed previously. This complexity is reflected in the power spectrum of the optimized electric field shown in Fig. 8c, which contains a multitude of peaks. Finally, as a check on the fidelity of the results, Fig. 8d shows that the norm-square of the propagated wavefunction, $\left|\psi_{N-1}\right|^{2}$, closely matches the desired target $\left|\psi_{f}\right|^{2}$. Taken together, both this example and the Morse potential discussed previously demonstrate the robustness of the NIC-CAGE software package for handling different potentials, propagation times, and various user-defined parameters. 

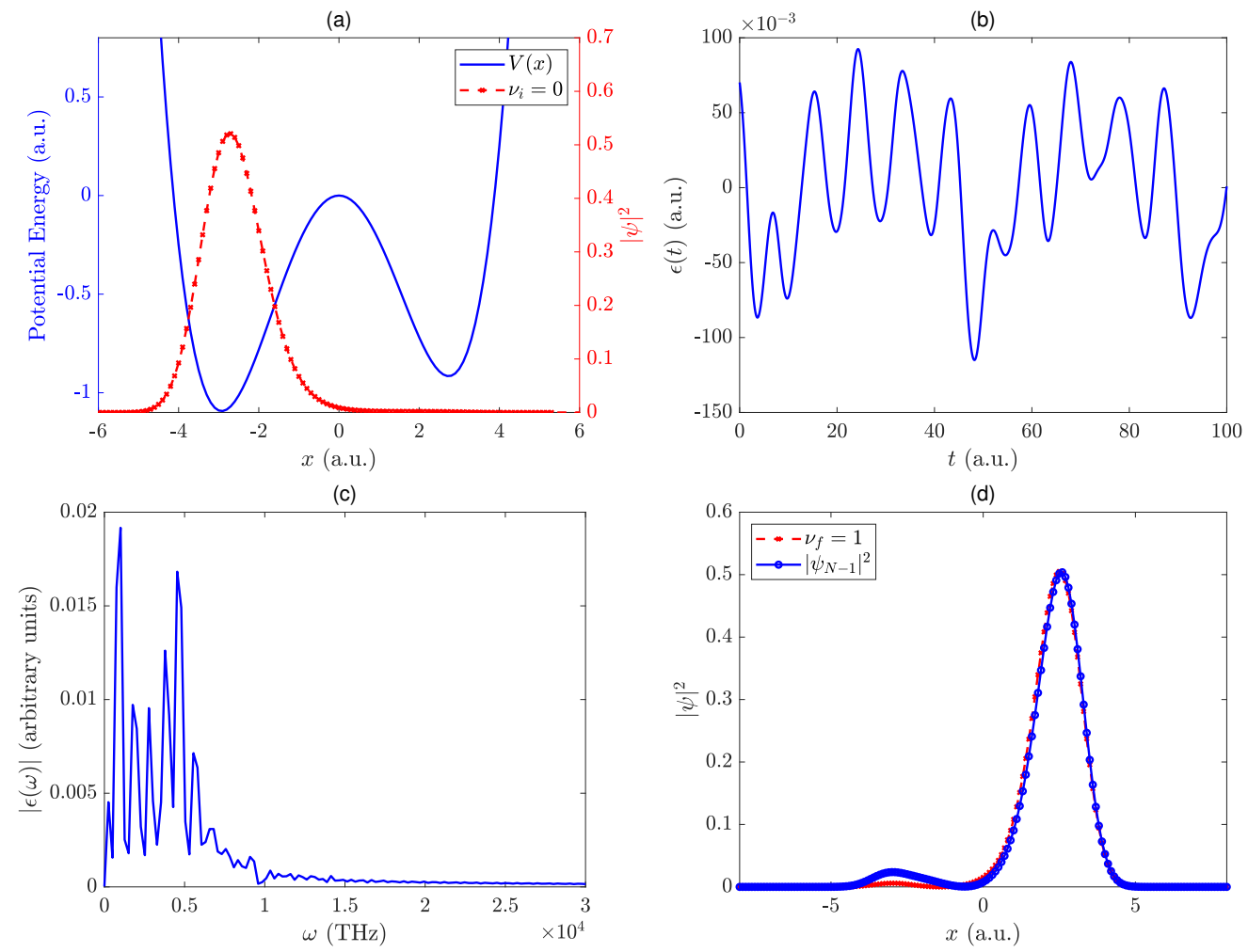

Figure 8: (a) Asymmetric double-well potential energy (solid blue line) and norm-squared initial vibrational eigenstate, $\left|\psi_{i}(x)\right|^{2}$, with $\nu_{i}=0$ (red dashed line). The numerical value of the potential energy and probability density can be measured from the left and right vertical axes, respectively. (b) Optimized electric field as a function of time for the $\nu_{i}=0 \rightarrow \nu_{f}=1$ transition. (c) Power spectrum of the optimized electric field. (d) Normsquared final target wavefunction $\left|\psi_{f}(x)\right|^{2}$ with $\nu_{f}=1$ and the propagated wavefunction $\left|\psi_{N-1}\right|^{2}$, which achieves a transition probability of $P=0.975$.

\section{Conclusions}

In this work, we have fully described, documented, and provided the NIC-CAGE software package - an open-source code for predicting optimized electric fields, $\epsilon(t)$, to control photo-excited chemical systems. Specifically, the NIC-CAGE code utilizes analytic gradients with a grid-based (normconserving) Crank-Nicolson propagation scheme to optically drive a chemical system from a known initial vibrational eigenstate to another desired state. Our approach is implemented in the high-level MATLAB and Python programming languages to allow researchers to get a detailed "look under the 
hood" to understand how our approaches are numerically incorporated in practice. Most importantly, we have tested the NIC-CAGE software package on a variety of different potentials, propagation times, and user-defined parameters to demonstrate its robustness in obtaining transition probabilities with extreme (typically over 97\%) accuracy.

Looking forward, we anticipate that the NIC-CAGE software package could be used in a multitude of applications to control photo-excited chemical systems. For instance, as demonstrated in our asymmetric double-well example, the optimal electric field, $\epsilon(t)$, can be strongly dependent on the total propagation time $T$. As such, the flexibility in choosing $T$ provides an opportunity to use the NIC-CAGE program to examine how this parameter can control the underlying dynamics. For example, if a low-intensity electric field is desired, then a long propagation time $T$ should be chosen. Alternatively, one may desire a short propagation time to overcome rapid relaxation mechanisms in a system (such as intramolecular energy transfer) [38] or other experimental physical limitations. In the same vein of exploring

parameter space, one can also use the NIC-CAGE program to explore the effect of altering the parameter, $\alpha$, in the fluence penalty term (cf. Eq. (7)). For instance, if a low-power electric field is desired, a larger value $\alpha$ should be chosen to penalize high pulse intensities and meet the experimental limitations of the user. Similarly, one can also modify $\alpha$ to have the form of a shape function such as a Gaussian pulse shape, which may be more experimentally accessible.

Finally, the NIC-CAGE software package could also be used as a predictive tool by experimentalists to give rigorous bounds on the wavelengths of light that would lead to the desired products in a photo-induced reaction/experiment. More concretely, an experimentalist could run this easyto-use code to predict the specific range of optical frequencies that would be needed to populate a desired photocatalytic product. We envision that further interactions of this kind between quantum control theorists and experimentalists would lead to further advances in site-specific excitations and bond-selective control in a multitude of photocatalytic and photo-excited systems.

\section{Acknowledgements}

This material is based upon work supported by the National Science Foundation under Grant No. CBET-1833218. This work used the Extreme 
Science and Engineering Discovery Environment (XSEDE) Comet computing cluster at the University of California, San Diego, through allocation TGENG160024.

\section{Author Contributions}

${ }^{\dagger}$ A.R. and C.H. contributed equally to this work. A.R. wrote the manuscript and carried out the quantum control calculations. C.H. and C.R.S. derived the initial gradient equations and wrote the initial software implementation. X.W., A.K., and B.M.W. derived the final gradient equations and finalized the software implementation. C.R.S. and B.M.W. supervised the work and wrote the manuscript.

\section{References}

[1] A. E. London, H. Chen, M. A. Sabuj, J. Tropp, M. Saghayezhian, N. Eedugurala, B. A. Zhang, Y. Liu, X. Gu, B. M. Wong, N. Rai, M. K. Bowman, J. D. Azoulay, A high-spin ground-state donor-acceptor conjugated polymer, Science Advances 5 (5). doi:10.1126/sciadv.aav2336.

URL https://advances . sciencemag .org/content/5/5/eaav2336

[2] D. B. Sulas, A. E. London, L. Huang, L. Xu, Z. Wu, T. N. Ng, B. M. Wong, C. W. Schlenker, J. D. Azoulay, M. Y. Sfeir, Preferential charge generation at aggregate sites in narrow band gap infrared photoresponsive polymer semiconductors, Advanced Optical Materials 6 (7) (2018) 1701138. doi:10.1002/adom. 201701138. URL https://onlinelibrary.wiley.com/doi/abs/10.1002/adom. 201701138

[3] J. D. Azoulay, Z. A. Koretz, B. M. Wong, G. C. Bazan, Bridgehead imine substituted cyclopentadithiophene derivatives: An effective strategy for band gap control in donoracceptor polymers, Macromolecules 46 (4) (2013) 1337-1342. doi:10.1021/ma302569u. URL https://doi .org/10.1021/ma302569u

[4] N. V. Ilawe, M. B. Oviedo, B. M. Wong, Real-time quantum dynamics of long-range electronic excitation transfer in plasmonic nanoantennas, Journal of Chemical Theory and Computation 13 (8) (2017) 3442-3454, pMID: 28679057. doi:10.1021/acs.jctc.7b00423. URL https ://doi.org/10.1021/acs.jctc.7b00423 
[5] N. V. Ilawe, M. B. Oviedo, B. M. Wong, Effect of quantum tunneling on the efficiency of excitation energy transfer in plasmonic nanoparticle chain waveguides, J. Mater. Chem. C 6 (2018) 5857-5864. doi:10. 1039/C8TC01466C.

URL http://dx.doi .org/10.1039/C8TC01466C

[6] L. Gunnarsson, T. Rindzevicius, J. Prikulis, B. Kasemo, M. Kll, S. Zou, G. C. Schatz, Confined plasmons in nanofabricated single silver particle pairs: experimental observations of strong interparticle interactions, The Journal of Physical Chemistry B 109 (3) (2005) 1079-1087, pMID: 16851063. doi:10.1021/jp049084e. URL https://doi .org/10.1021/jp049084e

[7] A. Naldoni, V. M. Shalaev, M. L. Brongersma, Applying plasmonics to a sustainable future, Science 356 (6341) (2017) 908-909. doi:10.1126/ science.aan5802. URL https://science.sciencemag.org/content/356/6341/908

[8] J. S. Jang, H. G. Kim, J. S. Lee, Heterojunction semiconductors: A strategy to develop efficient photocatalytic materials for visible light water splitting, Catalysis Today 185 (1) (2012) 270 - 277, catalysis on Energy and Environmental Technologies: 13th Korea-Japan Symposium on Catalysis. doi:https://doi.org/10.1016/j.cattod.2011.07.008.

URL http://www.sciencedirect.com/science/article/pii/ S092058611100527X

[9] K. Li, X. An, K. H. Park, M. Khraisheh, J. Tang, A critical review of $\mathrm{CO}_{2}$ photoconversion : Catalystsandreactors, Catalysis Today 224 (2014) 3 - 12, photocatalytic Processes for Environmental Remediation, in honor of Prof. Jincai Zhao of the Chinese Academy of Sciences. doi:https://doi.org/10.1016/j.cattod.2013.12.006.

URL http://wwW.sciencedirect.com/science/article/pii/ S0920586113006652

[10] X. Chen, S. Shen, L. Guo, S. S. Mao, Semiconductor-based photocatalytic hydrogen generation, Chemical Reviews 110 (11) (2010) 65036570, pMID: 21062099. doi:10.1021/cr1001645.

URL https://doi .org/10.1021/cr1001645 
[11] W. Zhu, J. Botina, H. Rabitz, Rapidly convergent iteration methods for quantum optimal control of population, The Journal of Chemical Physics 108 (5) (1998) 1953-1963. doi:10.1063/1.475576.

URL https://doi.org/10.1063/1.475576

[12] A. P. Peirce, M. A. Dahleh, H. Rabitz, Optimal control of quantummechanical systems: Existence, numerical approximation, and applications, Phys. Rev. A 37 (1988) 4950-4964. doi:10.1103/PhysRevA.37. 4950 .

URL https://link.aps.org/doi/10.1103/PhysRevA.37.4950

[13] P. Brumer, M. Shapiro, Coherence chemistry: controlling chemical reactions [with lasers], Accounts of Chemical Research 22 (12) (1989) 407413. doi:10.1021/ar00168a001.

URL https://doi .org/10.1021/ar00168a001

[14] S. Shi, H. Rabitz, Quantum mechanical optimal control of physical observables in microsystems, The Journal of Chemical Physics 92 (1) (1990) 364-376. doi:10.1063/1.458438.

URL https://doi.org/10.1063/1.458438

[15] E. B. Wilson, J. C. Decius, P. C. Cross, Molecular Vibrations: the Theory of Infrared and Raman Vibrational Spectra, Dover books on chemistry, Dover Publications, New York, NY, 1955.

[16] B. M. Wong, R. L. Thom, R. W. Field, Accurate inertias for largeamplitude motions: Improvements on prevailing approximations, The Journal of Physical Chemistry A 110 (23) (2006) 7406-7413, pMID: 16759129. doi:10.1021/jp057504+. URL https://doi.org/10.1021/jp057504+

[17] M. B. Oviedo, B. M. Wong, Real-time quantum dynamics reveals complex, many-body interactions in solvated nanodroplets, Journal of Chemical Theory and Computation 12 (4) (2016) 1862-1871, pMID: 26918732. doi:10.1021/acs.jctc.5b01019.

URL https ://doi.org/10.1021/acs.jctc.5b01019

[18] B. M. Wong, A. H. Steeves, R. W. Field, Electronic signatures of large amplitude motions: Dipole moments of vibrationally excited localbend and local-stretch states of s0 acetylene, The Journal of Physical Chemistry B 110 (38) (2006) 18912-18920, pMID: 16986883. doi: 
10.1021/jp061924x.

URL https://doi.org/10.1021/jp061924x

[19] K. Fukui, Formulation of the reaction coordinate, The Journal of Physical Chemistry 74 (23) (1970) 4161-4163. doi:10.1021/j100717a029.

URL https://doi .org/10.1021/j100717a029

[20] K. Fukui, The path of chemical reactions - the irc approach, Accounts of Chemical Research 14 (12) (1981) 363-368. doi:10.1021/ar00072a001. URL https : //doi .org/10.1021/ar00072a001

[21] B. M. Wong, S. Raman, Thermodynamic calculations for molecules with asymmetric internal rotorsapplication to 1,3-butadiene, Journal of Computational Chemistry 28 (4) (2007) 759-766. doi:10.1002/jcc. 20536. URL https://onlinelibrary.wiley.com/doi/abs/10.1002/jcc. 20536

[22] B. M. Wong, M. M. Fadri, S. Raman, Thermodynamic calculations for molecules with asymmetric internal rotors. ii. application to the 1,2-dihaloethanes, Journal of Computational Chemistry 29 (3) (2008) 481-487. doi:10.1002/jcc.20807.

URL https://onlinelibrary.wiley.com/doi/abs/10.1002/jcc. 20807

[23] H. Bechtel, A. Steeves, B. Wong, R. Field, Evolution of chemical bonding during honhnc isomerization as revealed through nuclear quadrupole hyperfine structure, Angewandte Chemie International Edition 47 (16) (2008) 2969-2972. doi:10.1002/anie.200705399.

URL https://onlinelibrary.wiley.com/doi/abs/10.1002/anie. 200705399

[24] B. M. Wong, Nuclear quadrupole hyperfine structure in hc14n/h14nc and dc15n/d15nc isomerization: a diagnostic tool for characterizing vibrational localization, Phys. Chem. Chem. Phys. 10 (2008) 5599-5606. doi:10.1039/B807672C.

URL http://dx.doi.org/10.1039/B807672C

[25] K. Prozument, R. G. Shaver, M. A. Ciuba, J. S. Muenter, G. B. Park, J. F. Stanton, H. Guo, B. M. Wong, D. S. Perry, R. W. Field, A new approach toward transition state spectroscopy, Faraday Discuss. 163 
(2013) 33-57. doi:10.1039/C3FD20160K.

URL http://dx.doi.org/10.1039/C3FD20160K

[26] D. P. Tew, N. C. Handy, S. Carter, S. Irle, J. Bowman, The internal coordinate path hamiltonian; application to methanol and malonaldehyde, Molecular Physics 101 (23-24) (2003) 3513-3525. doi: 10.1080/0026897042000178079. URL https : //doi .org/10.1080/0026897042000178079

[27] G. Reinisch, K. Miki, G. L. Vignoles, B. M. Wong, C. S. Simmons, An efficient and accurate formalism for the treatment of large amplitude intramolecular motion, Journal of Chemical Theory and Computation 8 (8) (2012) 2713-2724, pMID: 22904694. doi:10.1021/ct300278x. URL https : //doi .org/10.1021/ct300278x

[28] M. J. Frisch, G. W. Trucks, H. B. Schlegel, G. E. Scuseria, M. A. Robb, J. R. Cheeseman, G. Scalmani, V. Barone, G. A. Petersson, H. Nakatsuji, X. Li, M. Caricato, A. V. Marenich, J. Bloino, B. G. Janesko, R. Gomperts, B. Mennucci, H. P. Hratchian, J. V. Ortiz, A. F. Izmaylov, J. L. Sonnenberg, D. Williams-Young, F. Ding, F. Lipparini, F. Egidi, J. Goings, B. Peng, A. Petrone, T. Henderson, D. Ranasinghe, V. G. Zakrzewski, J. Gao, N. Rega, G. Zheng, W. Liang, M. Hada, M. Ehara, K. Toyota, R. Fukuda, J. Hasegawa, M. Ishida, T. Nakajima, Y. Honda, O. Kitao, H. Nakai, T. Vreven, K. Throssell, J. A. Montgomery, Jr., J. E. Peralta, F. Ogliaro, M. J. Bearpark, J. J. Heyd, E. N. Brothers, K. N. Kudin, V. N. Staroverov, T. A. Keith, R. Kobayashi, J. Normand, K. Raghavachari, A. P. Rendell, J. C. Burant, S. S. Iyengar, J. Tomasi, M. Cossi, J. M. Millam, M. Klene, C. Adamo, R. Cammi, J. W. Ochterski, R. L. Martin, K. Morokuma, O. Farkas, J. B. Foresman, D. J. Fox, Gaussian16 Revision C.01, gaussian Inc. Wallingford CT (2016).

[29] Y. Shao, Z. Gan, E. Epifanovsky, A. T. Gilbert, M. Wormit, J. Kussmann, A. W. Lange, A. Behn, J. Deng, X. Feng, D. Ghosh, M. Goldey, P. R. Horn, L. D. Jacobson, I. Kaliman, R. Z. Khaliullin, T. Ku, A. Landau, J. Liu, E. I. Proynov, Y. M. Rhee, R. M. Richard, M. A. Rohrdanz, R. P. Steele, E. J. Sundstrom, H. L. W. III, P. M. Zimmerman, D. Zuev, B. Albrecht, E. Alguire, B. Austin, G. J. O. Beran, Y. A. Bernard, E. Berquist, K. Brandhorst, K. B. Bravaya, S. T. Brown, D. Casanova, C.-M. Chang, Y. Chen, S. H. Chien, K. D. Closser, D. L. Crittenden, 
M. Diedenhofen, R. A. D. Jr., H. Do, A. D. Dutoi, R. G. Edgar, S. Fatehi, L. Fusti-Molnar, A. Ghysels, A. Golubeva-Zadorozhnaya, J. Gomes, M. W. Hanson-Heine, P. H. Harbach, A. W. Hauser, E. G. Hohenstein, Z. C. Holden, T.-C. Jagau, H. Ji, B. Kaduk, K. Khistyaev, J. Kim, J. Kim, R. A. King, P. Klunzinger, D. Kosenkov, T. Kowalczyk, C. M. Krauter, K. U. Lao, A. D. Laurent, K. V. Lawler, S. V. Levchenko, C. Y. Lin, F. Liu, E. Livshits, R. C. Lochan, A. Luenser, P. Manohar, S. F. Manzer, S.-P. Mao, N. Mardirossian, A. V. Marenich, S. A. Maurer, N. J. Mayhall, E. Neuscamman, C. M. Oana, R. Olivares-Amaya, D. P. ONeill, J. A. Parkhill, T. M. Perrine, R. Peverati, A. Prociuk, D. R. Rehn, E. Rosta, N. J. Russ, S. M. Sharada, S. Sharma, D. W. Small, A. Sodt, T. Stein, D. Stck, Y.-C. Su, A. J. Thom, T. Tsuchimochi, V. Vanovschi, L. Vogt, O. Vydrov, T. Wang, M. A. Watson, J. Wenzel, A. White, C. F. Williams, J. Yang, S. Yeganeh, S. R. Yost, Z.-Q. You, I. Y. Zhang, X. Zhang, Y. Zhao, B. R. Brooks, G. K. Chan, D. M. Chipman, C. J. Cramer, W. A. G. III, M. S. Gordon, W. J. Hehre, A. Klamt, H. F. S. III, M. W. Schmidt, C. D. Sherrill, D. G. Truhlar, A. Warshel, X. Xu, A. Aspuru-Guzik, R. Baer, A. T. Bell, N. A. Besley, J.-D. Chai, A. Dreuw, B. D. Dunietz, T. R. Furlani, S. R. Gwaltney, C.-P. Hsu, Y. Jung, J. Kong, D. S. Lambrecht, W. Liang, C. Ochsenfeld, V. A. Rassolov, L. V. Slipchenko, J. E. Subotnik, T. V. Voorhis, J. M. Herbert, A. I. Krylov, P. M. Gill, M. Head-Gordon, Advances in molecular quantum chemistry contained in the q-chem 4 program package, Molecular Physics 113 (2) (2015) 184-215. doi:10.1080/00268976.2014.952696. URL https://doi.org/10.1080/00268976.2014.952696

[30] M. W. Schmidt, K. K. Baldridge, J. A. Boatz, S. T. Elbert, M. S. Gordon, J. H. Jensen, S. Koseki, N. Matsunaga, K. A. Nguyen, S. Su, T. L. Windus, M. Dupuis, J. A. Montgomery Jr, General atomic and molecular electronic structure system, Journal of Computational Chemistry 14 (11) (1993) 1347-1363. doi:10.1002/jcc.540141112.

URL https://onlinelibrary.wiley.com/doi/abs/10.1002/jcc. 540141112

[31] M. Valiev, E. Bylaska, N. Govind, K. Kowalski, T. Straatsma, H. V. Dam, D. Wang, J. Nieplocha, E. Apra, T. Windus, W. de Jong, Nwchem: A comprehensive and scalable open-source solution for large scale molecular simulations, Computer Physics Communications 181 (9) (2010) 
1477 - 1489. doi:https://doi.org/10.1016/j.cpc.2010.04.018.

URL http://wWw.sciencedirect.com/science/article/pii/ S0010465510001438

[32] C. J. Joachain, N. J. Kylstra, R. M. Potvliege, Numerical integration of the wave equations, Cambridge University Press, 2011, p. 218266. doi:10.1017/CB09780511993459.006.

[33] W. Wirtinger, Zur formalen theorie der funktionen von mehr komplexen vernderlichen, Mathematische Annalen 97 (1927) 357-376.

URL http: //eudml .org/doc/182642

[34] G. von Winckel, A. Borzì, Computational techniques for a quantum control problem withH1-cost, Inverse Problems 24 (3) (2008) 034007. doi:10.1088/0266-5611/24/3/034007. URL https : //doi .org/10.1088\%2F0266-5611\%2F24\%2F3\%2F034007

[35] M. Sprengel, G. Ciaramella, A. Borz, A cokosnut code for the control of the time-dependent kohnsham model, Computer Physics Communications 214 (2017) 231 - 238. doi:https: //doi.org/10.1016/j.cpc.2017.01.020. URL http://wwW.sciencedirect.com/science/article/pii/ S0010465517300322

[36] W. H. Press, S. A. Teukolsky, W. T. Vetterling, B. P. Flannery, Numerical Recipes 3rd Edition: The Art of Scientific Computing, 3rd Edition, Cambridge University Press, New York, NY, USA, 2007, pp. 1024-1031.

[37] G. Paramonov, Coherent control of linear and nonlinear multiphoton excitation of molecular vibrations, Chemical Physics 177 (1) (1993) 169 - 180. doi:https://doi.org/10.1016/0301-0104(93)80187-E. URL http://www.sciencedirect.com/science/article/pii/ $030101049380187 \mathrm{E}$

[38] S. Shi, H. Rabitz, Optimal control of bond selectivity in unimolecular reactions, Computer Physics Communications 63 (1) (1991) $71-83$. doi:https://doi.org/10.1016/0010-4655(91)90239-H. URL http://www.sciencedirect.com/science/article/pii/ 001046559190239H 